6-10-2013

\title{
Conical Indentation of a Viscoelastic Sphere
}

\author{
J. K. Phadikar \\ University of Delaware
}

T. A. Bogetti

U.S. Army Research Laboratory

V. N. Kaliakin

University of Delaware

Anette M. Karlsson

Cleveland State University, a.karlsson@csuohio.edu

Follow this and additional works at: https://engagedscholarship.csuohio.edu/enme_facpub

Part of the Mechanical Engineering Commons

How does access to this work benefit you? Let us know!

\section{Original Citation}

Phadikar, J. K., Bogetti, T. A., Kaliakin, V. N., 2013, "Conical Indentation of a Viscoelastic Sphere," Journal of Engineering Materials and Technology, 135(4) pp. 041001.

This Article is brought to you for free and open access by the Mechanical Engineering Department at EngagedScholarship@CSU. It has been accepted for inclusion in Mechanical Engineering Faculty Publications by an authorized administrator of EngagedScholarship@CSU. For more information, please contact library.es@csuohio.edu. 


\title{
J. K. Phadikar \\ Department of Mechanical Engineering, \\ University of Delaware, \\ Newark, DE 19716 \\ T. A. Bogetti \\ U.S. Army Research Laboratory, \\ Conical Indentation of a Viscoelastic Sphere
} Aberdeen Proving Ground, MD 21001

V. N. Kaliakin

Department of Civil and

Environmental Engineering,

University of Delaware,

Newark, DE 19716

\author{
A. M. Karlsson ${ }^{1}$ \\ Department of Mechanical Engineering, \\ University of Delaware, \\ Newark, DE 19716: \\ Fenn College of Engineering \\ Cleveland State University, \\ Cleveland, $\mathrm{OH}$ 44115-2214 \\ e-mail: a.karlsson@csuohio.edu
}

Instrumented indentation is commonly used for determining mechanical properties of a range of materials, including viscoelastic materials. However, most-if not all—studies are limited to a flat substrate being indented by various shaped indenters (e.g., conical or spherical). This work investigates the possibility of extending instrumented indentation to nonflat viscoelastic substrates. In particular, conical indentation of a sphere is investigated where a semi-analytical approach based on "the method of functional equations" has been developed to obtain the force-displacement relationship. To verify the accuracy of the proposed methodology selected numerical experiments have been performed and good agreement was obtained. Since it takes significantly less time to obtain forcedisplacement relationships using the proposed method compared to conducting full finite element simulations, the proposed method is an efficient substitute of the finite element method in determining material properties of viscoelatic spherical particles using indentation testing.

\section{Introduction}

Techniques for evaluating materials with instrumented indentation have been developed over the past two decades and have emerged as a versatile tool to measure mechanical properties at micro or nanolevels, see for example, Refs. [1-5]. During an indentation experiment, a rigid indenter penetrates normally into a homogeneous solid and the indenter force, $P$, and depth of penetration, $h$, are continuously measured during a complete cycle of loading and unloading. A typical force-displacement curve is shown in Fig. 1(a). To extract the mechanical properties of a material from the force-displacement curve obtained from an indentation experiment, a clear understanding of the relationships between mechanical properties and various shape factors of the force-displacement curve need to be developed. Computational and theoretical studies are typically used to elucidate the contact mechanics and deformation mechanisms so to systematically extract mechanical properties from force-displacement relationship [1-5].

In this work, we are investigating indentation of small ${ }^{2}$ spherical structures, made of visco-elastic material. Examples of smallscale spherical bodies requiring material characterization include micron-sized metal coated polymer particles used in the manufacturing of anisotropic conductive adhesives [6,7], polymer latex particles for controlling the mechanical properties of latex films $[8,9]$ used in synthetic latex materials, and living cells [10].

Although most of the literature discussing indentation techniques is devoted to time-independent elastic-plastic materials, the indentation technique has recently been applied to time-dependent materials, see, for example, Refs. [11-15]. Cheng et al. [11] used "the method of functional equations" [16] to develop viscoelastic indentation solutions for spherical indentation on a semi-infinite half space. Similarly, Vandamme and Ulm [12] developed

\footnotetext{
Corresponding author

"Small" is relative to the indentation depth; that is, the indentation depth affects the overall behavior of the sphere and cannot be considered to be local around the indentation.

Contributed by the Materials Division of ASME for publication in the JournaL of Engineering Materials and Technology. Manuscript received January 10, 2013; final manuscript received April 21, 2013; published online June 10, 2013. Assoc. Editor: Georges Cailletaud.
}

analytical solutions for viscoelastic conical indentation on a semiinfinite half-space. Francius et al. [13] determined the viscoelastic properties of polyelectrolyte multilayer films using a standard linear solid model. Cheng and Cheng [14] established a relationship between initial unloading slope, contact depth and the instantaneous modulus for sufficiently high rate of unloading for conical indentation on a viscoelastic half-space.

Most work pertaining to viscoelastic indentation has been dedicated to indentation of flat surfaces [11-14]. Zhou and Lu [15] investigated viscoelastic properties of a spherical object indented by a spherical indenter tip. However, using Hertz-type solutions [1], the procedure is limited to shallow indentation depths.

In this paper, we investigate the indentation of a viscoelastic sphere by a conical indenter as shown in Fig. 1(b), with the ultimate goal of developing a reliable evaluation technique for indentation testing of viscoelastic spherical particles. For this purpose, a semi-analytical technique has been employed for the forward analysis of the indentation problem, which is then verified by a geometrically nonlinear finite element analysis.

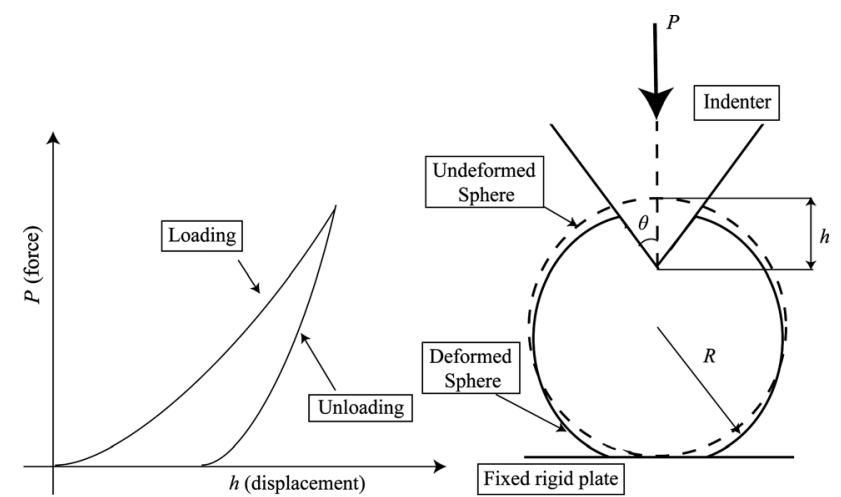

(a)

(b)

Fig. 1 (a) Force-displacement relationship of a typical indentation experiment and $(b)$ conical indentation of a sphere resting on a rigid and flat surface 


\section{Semi-Analytical Approach}

2.1 Elastic Solution. Lee and Radok [16] developed the method of functional equations for solving viscoelastic indentation problems using a corresponding linear-elasticity solution. In this approach, if the elastic solution of a boundary value problem is known, the functional equations can be obtained by replacing the elastic constants in the solution by equivalent viscoelastic operators. Here, we determine the elastic solution of the conesphere contact problem by geometrically nonlinear finite element analysis. For a particular indenter half-angle, $\theta$, the indenter force, $P$, is in general a function of the indentation depth, $h$, the radius of the sphere, $R$, the elastic modulus, $E$, and Poisson's ratio, $\nu$, of the material. Thus

$$
P=f_{1}(E, h, R, \nu) \leftarrow
$$

Applying dimensional analysis and utilizing Buckingham's PI theorem [17], the above equation can be written in its nondimensional form as follows:

$$
\frac{P}{E R^{2}}=f_{2}\left(\frac{h}{R}, \nu \psi(\right.
$$

We assume the following specific form of Eq. (2)

$$
\frac{P}{E_{\mathrm{r}} R^{2}}=\phi\left(\frac{h}{R}\right)
$$

In this equation, $E_{\mathrm{r}}$ is the plain strain reduced modulus, which is given by

$$
E_{\mathrm{r}}=\frac{E}{1-\nu^{2}}
$$

The validity of the above assumption, as well as the functional form of $\phi$, will be established using finite element analysis later in this paper. The reduced modulus, $E_{\mathrm{r}}$, generally appears in contact problems where contact interference is very small compared to the dimensions of the contacting bodies. However, it will be subsequently shown that even for relatively deep indentation depth, the elastic modulus and Poisson's ratio can be combined into the reduced modulus, $E_{\mathrm{r}}$.

\subsection{Viscoelastic Solution}

The viscoelastic behavior of a material can be represented by mechanical models consisting of finite networks of springs and dashpots. Here we simulate the behavior of a visoelastic material with a four-parameter Kelvin-Voigt deviator creep model [18] shown in Fig. 2(a). In this model, the shear behavior is represented by the standard three-element solid model (Figs 2(a) and $2(b)$ ) where $G_{1}$ and $G_{2}$ are the moduli of the two spring elements and $\eta$ is the viscosity of the dashpot element. The bulk behavior, with bulk modulus $K$ (Fig. 2(b)), is time independent.

To express the constitutive relation for this model, the stress and strain tensors need to be decomposed into deviatoric and spherical (volumetric) components as

$$
\begin{aligned}
& \sigma_{i j}=\sigma_{i j}^{D}+\sigma_{i j}^{V} \\
& \varepsilon_{i j}=\varepsilon_{i j}^{D}+\varepsilon_{i j}^{V}
\end{aligned}
$$

where indicial notation is employed, with $i, j=1,2,3$. The deviatoric and spherical components in terms of stress and strain tensors are given by

$$
\begin{aligned}
& \sigma_{i j}^{D}=\sigma_{i j}-\frac{1}{3} \sigma_{k k} \delta_{i j} ; \quad \sigma_{i j}^{V}=\frac{1}{3} \sigma_{k k} \delta_{i j} \\
& \varepsilon_{i j}^{D}=\sigma_{i j}-\frac{1}{3} \varepsilon_{k k} \delta_{i j} ; \quad \varepsilon_{i j}^{V}=\frac{1}{3} \varepsilon_{k k} \delta_{i j}
\end{aligned}
$$

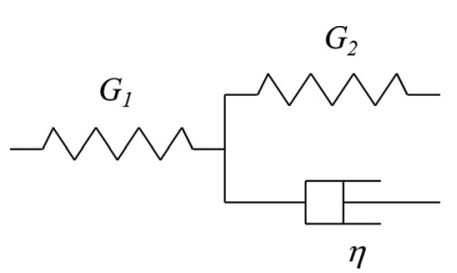

(a)

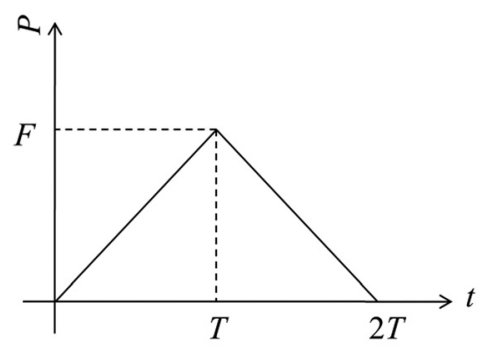

(c)
$K$ $\widehat{\Upsilon N}$

\section{Fig. 2 Assumed constitutive behavior of the viscoelastic mate- rial and the loading function: (a) standard three-element solid model for deviatoric behavior, $(b)$ spring element for spherical} (volumetric) behavior, and (c) triangular loading

where $\delta_{i j}$ is the Kronecker delta function. In the time, $t$, domain, the constitutive relation can be written as [18]

$$
\begin{gathered}
\sigma_{i j}^{D}+\frac{\eta}{G_{1}+G_{2}} \frac{d \sigma_{i j}^{D}}{d t}=\frac{G_{1} G_{2}}{G_{1}+G_{2}} 2 \varepsilon_{i j}^{D}+\frac{G_{1} \eta}{G_{1}+G_{2}} \frac{d\left(2 \varepsilon_{i j}^{D}\right)}{d t} \\
\sigma_{i j}^{V}=3 K \varepsilon_{i j}^{V}
\end{gathered}
$$

Employing Laplace transforms, the constitutive relations take the following form in the Laplace domain, using the Laplace integration parameter, $s$ [18]

$$
\begin{aligned}
& \sigma_{i j}^{D}(s)=2 G(s) \varepsilon_{i j}^{D}(s) \leftarrow \\
& \sigma_{i j}^{V}(s)=3 K(s) \varepsilon_{i j}^{V}(s) \leftarrow
\end{aligned}
$$

where

$$
\begin{gathered}
G(s)=\frac{G_{1} G_{2}+G_{1} \eta s}{G_{1}+G_{2}+\eta s} \\
K(s)=K
\end{gathered}
$$

Applying the "method of functional equations" [16] to the elastic solution given by Eq. (3), the following expression for the time-dependent indentation depth behavior under an arbitrary prescribed indentation loading history, $P(t)$, can be obtained

$$
\phi\left(\frac{h(t)}{R}\right)=\frac{1}{R^{2}} \int_{0}^{t} J(t-\xi) \underset{d P(\xi) \leftarrow}{d \xi}
$$

where $J(t)$ is the creep compliance function associated with the reduced modulus $E_{\mathrm{r}}$ and $\xi$ is the variable of integration. Eq. (8) can also be written in the Laplace domain as follows:

$$
\phi\left(\frac{h(t)}{R}\right)=\left(\frac{1}{R^{2}} \Gamma^{-1}\left(\frac{P(s)}{E_{\mathrm{r}}(s)}\right)\right.
$$

where $\Gamma^{-1}$ denotes inverse Laplace transform. Eq. (9) can easily be inverted to determine the indenter displacement as a function of time as follows: 


$$
h(t)=R \phi^{-1}\left[\frac{1}{R^{2}} \Gamma^{-1}\left(\frac{P(s)}{E_{\mathrm{r}}(s)}\right)\right] \quad(
$$

Thus, for a given radius of the sphere, loading history and constitutive equation, Eq. (10) can be used to determine the indenter displacement as a function of time. It follows that the forcedisplacement relationship for a specific viscoelastic indentation problem can thus be obtained. For the constitutive equation under consideration (Eq. (7)), the reduced modulus in the Laplace domain becomes (using Eqs. (7c) and (7d))

$$
\begin{aligned}
E_{\mathrm{r}}(s) & =\overleftarrow{E(s) \leftarrow}=\frac{4 G(s)\{3 K(s)+G(s)\}}{3 K(s)+4 G(s) \leftarrow} \Rightarrow E_{\mathrm{r}}(s) \\
& =4 G_{1} \frac{\left(G_{2}+s \eta\right)\left\{3 K\left(G_{1}+G_{2}+s \eta\right)+G_{1}\left(G_{2}+s \eta\right)\right\}}{\left(G_{1}+G_{2}+s \eta\right)\left\{3 K\left(G_{1}+G_{2}+s \eta\right)+4 G_{1}\left(G_{2}+s \eta\right)\right\}}
\end{aligned}
$$

To illustrate this, we consider loading-unloading history with maximum force $F$ and time period $2 T$ applied as a triangular ramp as shown in Fig. 2(c) as an example. The loading function for this history can be written as

$$
\begin{aligned}
P(t)= & \underset{T}{T} t[H(t)-H(t-T)]+\left\{-\frac{F}{T} t+2 F\right\} \\
& \times[H(t-T)-H(t-2 T)] \leftarrow
\end{aligned}
$$

where $H(t)$ is the Heaviside function. Taking the Laplace transform of Eq. (12) gives

$$
P(s)=\frac{F}{T s^{2}}\{1-2 \exp (-T s)+\exp (2 T s)\} \leftarrow
$$

Substituting $E_{\mathrm{r}}(s)$ from Eq. (11), and $P(s)$ from Eq. (13) into Eq. (10), we obtain

$$
\begin{aligned}
h(t)= & R \phi^{-1}\left[\frac { 1 } { R ^ { 2 } } \Gamma ^ { - 1 } \left(\frac{F\{1-2 \exp (-T s)+\exp (2 T s)\}}{4 T s^{2} G_{1}\left(G_{2}+s \eta\right) \leftarrow}\right.\right. \\
& \left.\left.\times \frac{\left(G_{1}+G_{2}+s \eta\right)\left\{3 K\left(G_{1}+G_{2}+s \eta\right)+4 G_{1}\left(G_{2}+s \eta\right)\right\}}{\left\{3 K\left(G_{1}+G_{2}+s \eta\right)+G_{1}\left(G_{2}+s \eta\right)\right\} \leftarrow}\right)\right]
\end{aligned}
$$

By determining the inverse Laplace transform in the above expression, the displacement of the indenter can be computed as a function of time. The force-displacement relationship of the indentation problem can then be obtained by combining the loading and displacement history.

In Sec. 3, we will consider a numerical approach to investigate the accuracy of the present semi-analytical approach.

\section{Finite Element Analysis}

The commercial finite element code ABAQUS [19] has been used to simulate the elastic indentation of the sphere by a conical indenter. The sphere is assumed composed of homogeneous, isotropic, elastic material. A two-dimensional axisymmetric model was adopted. The sphere was discretized by approximately 24,000 four-node (CAX4R) elements. The sphere is assumed to rest on a flat surface. Both the surface and the indenter are modeled as rigid bodies. Several simulations with refined meshes and smaller time increments were investigated as part of a convergence study. The model used, shown in Fig. 3, gave the same results as a finer mesh and time increment. The selected mesh density is thus sufficient to accurately simulate the mechanism of indentation. The surface nodes of the sphere are traction-free and the nodes along the axis of symmetry are constrained in the direction normal to indenter displacement so as to simulate symmetry conditions. The rigid

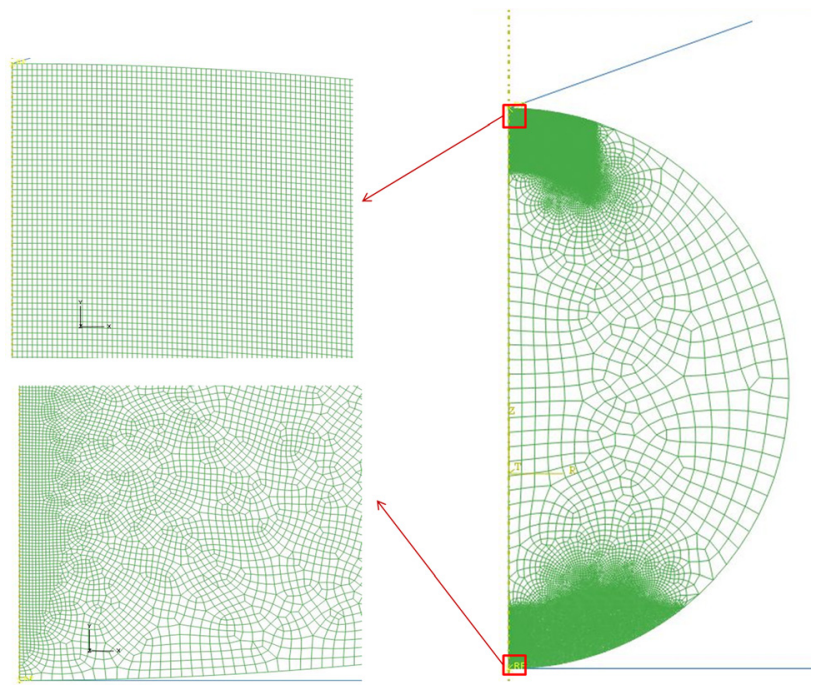

Fig. 3 Finite element model used in ABAQus, including enlargement of the refined mesh (plotted at the same scale) at the top of the sphere (conical indentation) and the bottom of the sphere (contact with the rigid surface) for the present indentation problem

surface at the bottom of the sphere is kept fixed in all three directions.

\section{Results}

A sphere of radius $R=23 \mu \mathrm{m}$ and made of a material with $E=1000 \mathrm{MPa}$ was numerically indented by a conical indenter with half-angle $\theta=70.3 \mathrm{deg}$. To examine the validity of the assumption made in Sec. 2.1, the force-displacement curves are plotted in Fig. 4 for four different Poisson's ratios up to a maximum indentation depth of $15 \%$ of the radius.

Clearly there is some difference between these curves. The same data have been represented in Fig. 5, where $P /\left(E_{\mathrm{r}} R^{2}\right)$ has been plotted as a function of $h / R$ (recall Eq. (3)) for four different Poisson's ratios.

Within the resolution of Fig. 5, the results obtained for the investigated Poison's ratio essentially overlap, thus validating the assumption of writing Eq. (2) as Eq. (3). By curve-fitting, function $\phi$ in Eq. (3) was determined to be

$$
\begin{aligned}
\phi\left(\frac{h}{R}\right)= & 4.42685\left(\frac{h}{R}\right)^{4}-2.07899\left(\frac{h}{R}\right)^{3}+0.932136\left(\frac{h}{R}\right)^{2} \\
& +0.00468643\left(\frac{h}{R}\right)-0.0000102475
\end{aligned}
$$

The function $\phi$ can be inverted and used in Eq. (14) to determine the force-displacement relationship of the viscoelastic indentation problem. Since $\phi$ is a fourth order polynomial, Eq. (14) yields four values of the displacement, $h$, (for a given $t$, since $h$ is a function of $t$ ) and the realistic positive value was selected ignoring the negative and imaginary roots. The curve fitting as well as other computations involved in solving Eq. (14) to determine the displacement, $h$, were performed using the commercial software MATLAB [20]. The method is characterized as semi-analytical since it uses finite element simulations to obtain the functional form of $\phi$ (Eq. (14)).

As an example, we considered a sphere of radius $23 \mu \mathrm{m}$ indented by an indenter of half-angle $70.3 \mathrm{deg}$. Following the simulations conducted in Refs. [2,12], the viscoelastic material properties are assumed as $G_{1}=234.6 \mathrm{MPa}, G_{2}=25.78 \mathrm{MPa}$, $\eta=257.78$ and $K=687.62 \mathrm{MPa}$. Four values of loading time $T$ 


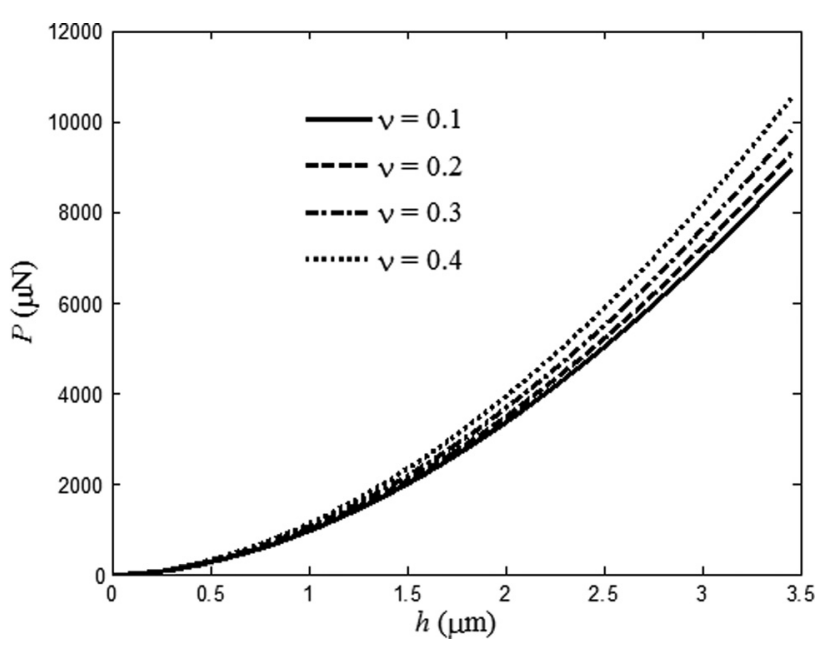

Fig. 4 The force-displacement relationships for the elastic indentation problem, for selected Poisson's ratios as obtained from geometrically nonlinear finite element analysis

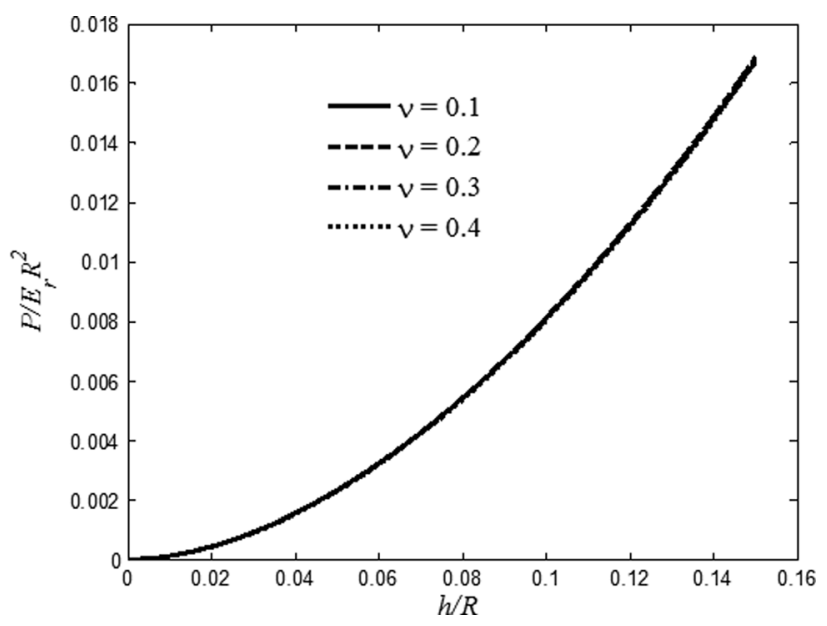

Fig. 5 Normalized force-displacement relationships for the elastic indentation problem, for four selected Poisson's ratios

was selected: $T=1 \mathrm{~s}, T=10 \mathrm{~s}, T=20 \mathrm{~s}$, and $T=30 \mathrm{~s}$; and all cases are loaded up to maximum force, $F=1000 \mu \mathrm{N}$. The forcedisplacement relationships obtained using the proposed semianalytical approach are plotted in Fig. 6.

To verify the semi-analytical approach for the indentation of a viscoelastic sphere discussed above, we used ABAQus to simulate the problem numerically. The finite element model is the same as that described in Sec. 3 except that the viscoelastic material properties, in form of Prony series, have been used instead of elastic properties. The force-displacement relationships thus obtained for the indentation problem with geometry and material properties described in Sec. 3.1 are plotted in Fig. 6.

It can be seen from Fig. 6 that the semi-analytical approach captures the loading curve quite accurately. It is noted that as the load time, $T$, increases, the difference between the semi-analytical model and the direct visco-elastic analysis increases (the error at $\max$ load for $T=1 \mathrm{~s}$ is $0.57 \%$ and for $T=30 \mathrm{~s}$ is $1.62 \%$ ). This may be due to the increase in inaccuracy of the regression used in Eq. (15), with increasing depth-to-radius ratio. The increase in error in maximum displacement with load time was observed in a previous work [12] as well and the authors suggested the inaccuracy of the elastic solution as a possible reason.

The semi-analytical approach captures the initial unloading well, similarly as to loading. However, it does not capture the final unloading part accurately. This was also observed for the case of

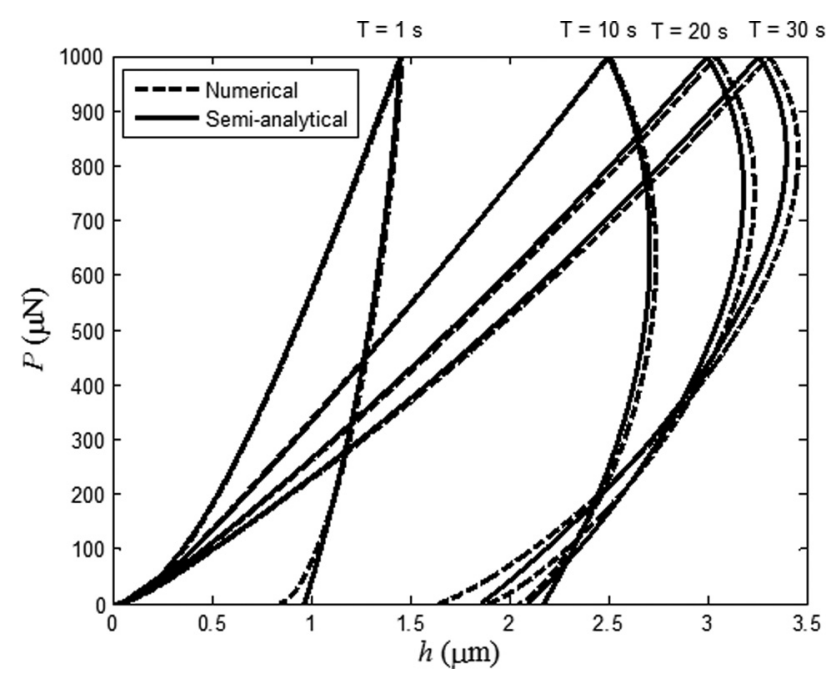

Fig. 6 Comparison of force-displacement curves obtained using the proposed semi-analytical approach and ABAQus for four selected loading times, $T$

conical indentation on viscoelastic half-space [12]. In that case, the authors concluded that the discrepancy is due to that the method of functional equations is valid only when the contact radius is a monotonically increasing function of time (as reported by the researchers [16] who developed the method), which is not true during the final part of unloading.

However, there is a significant difference, between the computational costs involved in obtaining the force-displacement relationships using the semi-analytical method and the direct finite element simulations. For example, for the case $T=30 \mathrm{~s}$, approximately 5 CPU hours were needed to obtain the force-displacement relationship using finite element simulation, whereas it took less than $1 \mathrm{CPU}$ second for the semi-analytical method (excluding the elastic finite element analysis). Computations were performed in a DELL Precision T7400 workstation with two Intel(R) Xeon(R) X5472@3 GHz processors. Thus, the semi-analytical method is a viable method for obtaining force-displacement relationships for viscoelastic spheres where those are required for various material properties and loading histories, for example, in determining the viscoelatic material properties of spherical particles using indentation tests. The material properties can be obtained from the force-displacement relationship using a suitable reverse analysis technique as commonly done for timeindependent materials [3-5]. Although, the method has been demonstrated for triangular loading-unloading history, the method can be applied for ramp-loading, hold, ramp-unloading history, which is commonly used in experiments pertaining to indentation of viscoelastic materials. This is illustrated for conical indentation on half-space in a previous work [12].

\section{Concluding Remarks}

A semi-analytical approach has been proposed to investigate the conical indentation of a viscoelastic sphere. The approach is based on "the method of functional equations" and uses the finite element simulation results for the elastic indentation of a sphere. For a specific viscoelastic material model and loading history, the results from the semi-analytical approach have been compared to the finite element simulation results of the indentation of a viscoelastic sphere. The results agree well for most of the loading and unloading parts. The proposed semi-analytical approach can easily be applied to other loading histories and viscoelastic material models. Thus, the proposed method can be useful for the determination of viscoelastic material properties of spherical particles based on an indentation test, since it is computationally much less intensive than the finite element simulations. 


\section{References}

[1] Johnson, K. L., 1987, Contact Mechanics, Cambridge University Press, Cambridge, UK.

[2] Cheng, Y. T., and Cheng, C. M., 2004, "Scaling, Dimensional Analysis, and Indentation Measurements," Mater. Sci. Eng. R, 44, pp. 91-149.

[3] Kucharski, S., and Mróz, Z., 2007, "Identification of Yield Stress and Plastic Hardening Parameters From a Spherical Indentation Test,” Int. J. Mech. Sci., 49, pp. 1238-1250.

[4] Yan, J., Karlsson, A. M., and Chen, X., 2007, "Determining Plastic Properties of a Material With Residual Stress by Using Conical Indentation," Int. J. Solids Struct., 44, pp. 3720-3737.

[5] Phadikar, J. K., Bogetti, T. A., and Karlsson, A. M., 2012, "On Establishing Elastic-Plastic Properties of a Sphere by Indentation Testing," Int. J. Solids Struct., 49, pp. 1961-1972.

[6] Kristiansen, H., Shen, Y., and Liu, J., 2001, "Characterisation of Mechanical Properties of Metal-Coated Polymer Spheres for Anisotropic Conductive Adhesive," First International IEEE Conference on Polymers and Adhesives in Microelectronics and Photonics (POLYTRONIC 2001), Potsdam, Germany, October 21-24, pp. 344-348.

[7] Kwon, W. S., and Paik, K. W., 2006, "Experimental Analysis of Mechanical and Electrical Characteristics of Metal-Coated Conductive Spheres for Anisotropic Conductive Adhesives (ACAs) Interconnection," IEEE Trans. Compon. Packag. Technol., 29, pp. 528-534.

[8] Misawa, H., Koshioka, M., Sasaki, K., Kitamura, N., and Masuhara, H., 1991, "Three Dimensional Optical Trapping and Laser Ablation of a Single Polymer Latex Particle in Water," J. Appl. Phys., 70, pp. 3829-3836.
[9] Tamai, H., Hasegawa, M., and Suzawa, T., 1989, "Surface Characterization of Hydrophilic Functional Polymer Latex Particles,” J. Appl. Polym. Sci., 38, pp. 403-412.

[10] Dao, M., Lim, C., and Suresh, S., 2003, "Mechanics of the Human Red Blood Cell Deformed by Optical Tweezers," J. Mech. Phys. Solids, 51, pp. 2259-2280.

[11] Cheng, L., Xia, X., Scriven, L. E., and Gerberich, W. W., 2005, "Spherical-Tip Indentation of Viscoelastic Material," Mech. Mater., 37, pp. 213-226.

[12] Vandamme, M., and Ulm, F. J., 2006, "Viscoelastic Solutions for Conical Indentation," Int. J. Solids Struct., 43, pp. 3142-3165.

[13] Francius, G., Hemmerlé, J., Ball, V., Lavalle, P., Picart, C., and Voegel, J. C., 2007, "Stiffening of Soft Polyelectrolyte Architectures by Multilayer Capping Evidenced by Viscoelastic Analysis of AFM Indentation Measurements," J. Phys. Chem. C., 111, pp. 8299-8306.

[14] Cheng, Y. T., and Cheng, C. M., 2005, "General Relationship Between Contact Stiffness, Contact Depth, and Mechanical Properties for Indentation in Linear Viscoelastic Solids Using Axisymmetric Indenters of Arbitrary Profiles," Appl. Phys. Lett., 87, p. 111914.

[15] Zhou, Z., and Lu, H., 2010, "On the Measurements of Viscoelastic Functions of a Sphere by Nanoindentation,” Mech. Time-Depend. Mater., 14, pp. 1-24.

[16] Lee, E. H., and Radok, J. R. M., 1960, "The Contact Problem for Viscoelastic Bodies," ASME J. Appl. Mech., 27, pp. 438-444.

[17] Buckingham, E., 1914, "On Physically Similar Systems, Illustrations of the Use of Dimensional Equations,” Phys. Rev., 4, pp. 345-376.

[18] Haddad, Y., 1995, Viscoelasticity of Engineering Materials, Springer, New York.

[19] Dassault Systèmes., 2009, ABAQus Theory Manual version 6.9-2.

[20] Matlab R2011a, 2010, Natick, The MathWorks, Inc., Natick, MA 